Service social

\title{
Habiletés propres au travail avec le groupe comme entité
}

\section{Ruth R. Middleman}

Volume 39, numéro 1, 1990

Le groupe ici et ailleurs

URI : https://id.erudit.org/iderudit/706462ar

DOI : https://doi.org/10.7202/706462ar

Aller au sommaire du numéro

\section{Éditeur(s)}

École de service social de l'Université Laval

\section{ISSN}

1708-1734 (numérique)

Découvrir la revue

\section{Citer cet article}

Middleman, R. R. (1990). Habiletés propres au travail avec le groupe comme entité. Service social, 39(1), 151-159. https://doi.org/10.7202/706462ar

\section{Résumé de l'article}

L'article traite des habiletés particulièrement utiles au travailleur social qui veut vraiment tirer profit des ressources d'un groupe en tant qu'entité. On y présente trois habiletés fondamentales pour toute intervention de groupe, puis seize habiletés plus ponctuelles, les unes davantage utiles durant la phase de formation du groupe, les autres surtout de nature à faciliter la réussite de la phase de travail du groupe. Une feuille de pointage est proposée en annexe, à titre d'outil pour faciliter la consignation de données d'observation. 


\section{COMMENTAIRES ET DOCUMENTS}

Ruth R. Middleman, professeure à l'École de service social Kent, Université de Louisville, Kentucky.

\section{Habiletés propres au travail avec le groupe comme entité}

Ruth R. Middleman

Le présent article traite de diverses habiletés particulièrement utiles au travailleur social qui veut vraiment tirer profit des ressources d'un groupe en tant qu'entité. On y discute d'abord de trois habiletés fondamentales que l'on devrait retrouver tout au long de l'intervention et dans tous les groupes. Seize autres habiletés plus ponctuelles sont aussi présentées, les unes davantage utiles durant la phase de formation du groupe, les autres surtout de nature à faciliter la réussite de la phase de travail du groupe. Une feuille de pointage est présentée, en annexe, à titre d'outil pour faciliter la consignation de données d'observation d'un groupe.

Nous examinons d'abord les habiletés plus fondamentales que doit utiliser le travailleur social de groupe, c'est-à-dire celles qui doivent se manifester de façon constante dans les groupes; elles réfèrent à des attitudes et comportements spécifiques au travail avec un petit groupe. La première, globale, sous-tend toutes les autres : elle consiste à penser en termes de groupe. La deuxième réfère à un comportement plus spécifique du travailleur social : balayer le groupe du regard. La troisième est directement liée à la vie du groupe : il s'agit de favoriser la cohésion des membres. 


\section{Penser en termes de groupe}

Penser en termes de groupe, $c^{\prime}$ est avoir bien en tête les concepts de groupe, ceux-ci devenant notre cadre de référence lorsque nous observons et analysons ce qui se passe dans le groupe. C'est une habileté cognitive. Penser en termes de groupe implique que les concepts de groupe prennent le dessus sur les concepts et la dynamique individuels ou relatifs à la personnalité, comme s'ils étaient un prisme qui permet de "voir » ce qui se passe. Le travailleur social utilise une "lentille à grand angle " pour comprendre les phénomènes de groupe puisqu'il sait que les clients ont, en groupe, des comportements différents de ceux qu'ils adoptent en tant qu'individus isolés.

La connaissance du groupe implique, entre autres, qu'il faille comprendre l'impact de certains concepts tels que : la taille, les rôles, les normes, la formation du groupe, les modèles de communication, le réseau d'attractions interpersonnelles, la mutualité, le leadership, I'homogénéité, la cohésion, l'influence et les étapes de développement du groupe. Ces réalités tiendront une place plus importante dans l'intervention que, par exemple, les valeurs personnelles, les modèles de comportement individuel, ou la réaction possible d'un participant à ce que d'autres membres expriment dans le groupe, puisque ces concepts sont associés aux puissantes forces induites par le contexte et la composition du groupe.

Penser en termes de groupe, cela signifie dans un premier temps, voir le groupe comme une entité et, ensuite, considérer les participants à titre individuel, lorsqu'ils initient une interaction ou répondent à celle d'un pair. L'intervenant relâche un peu le contrôle qu'il exerce et démontre qu'il saisit et comprend la coexistence de plusieurs relations aidantes ayant cours dans le groupe.

\section{Balayer du regard (scanning)}

Le balayage ou "scanning " consiste à embrasser du regard le groupe entier. L'intervenant promène son regard pour avoir un contact visuel avec tous les participants plutôt que de s'arrêter sur une ou deux personnes seulement. Ainsi, le travailleur social capte activement différents signaux de communication et signifie son intérêt pour chacun d'eux. Le balayage demande des habiletés de communication sensorielles, cognitives et interpersonnelles, puisque l'intervenant remarque $d^{\prime}$ 'une manière sélective ce que tous les participants du groupe expriment - de façon verbale ou non verbale - et démontre qu'il est en contact avec chacun d'eux.

Pour avoir une telle vue d'ensemble, l'intervenant promène son regard à travers le groupe, ne s'attarde sur personne en particulier, 
sans cependant effectuer de mouvements rapides ou mécaniques qui donneraient une impression de rigidité ou de routine. Le balayage devrait se faire de façon naturelle et engageante, et comporter un contact visuel et une attention qui expriment : " Je m'intéresse à vous tous ». Par cette technique, le travailleur social aide les membres du groupe à sentir qu'il est là pour tout le monde même lorsqu'une personne domine la communication.

\section{Favoriser la cohésion}

Les mots utilisés peuvent favoriser la cohésion. Lorsque l'intervenant parle au groupe, ou au sujet du groupe, il doit toujours dire "nous", " notre ", " nos", et jamais " je », "vous ", " votre ». Ce changement de vocabulaire peut paraitre bizarre au début, mais I'habitude s'installe rapidement et cela devient naturel. Par cette simple modification sémantique, l'intervenant exprime qu'il perçoit le groupe comme une entreprise d'ensemble et non pas comme une affaire de "vous" et de " eux ". Ceci en dit long sur la position que I'intervenant désire prendre face au groupe et à son processus.

L'utilisation d'habiletés plus ponctuelles, de nature à aider le groupe dans son développement et son fonctionnement, fait aussi partie des préoccupations du travailleur social. Nous en décrivons d'abord cinq, susceptibles d'aider à la formation du groupe.

\section{Choisir délibérément des modèles de communication}

Choisir délibérément un modèle de communication, $c^{\prime}$ est porter un jugement sur une façon de communiquer qu'on croit facilitante et qui correspond aux objectifs du groupe, à sa taille et à son étape de développement, et le mettre en pratique. Ceci peut se produire au début d'une expérience de groupe, au début d'une session, ou à tout moment au cours d'une réunion, si un changement de rythme ou de direction semble approprié et que les participants indiquent leur acquiescement à aller de l'avant de la façon proposée.

\section{Signaler les réalisations du groupe}

Signaler les réalisations du groupe, c'est résumer verbalement, en manifestant son appréciation, tout indice de progrès ou de croissance perçu par l'intervenant, que cela vienne du groupe ou d'une personne en particulier. Il peut s'agir d'actions, de pensées ou d'émotions dont les membres ont pris conscience ou pas. L'intervenant attire périodiquement l'attention sur des indices d'évolution, 
spécialement lorsqu'ont été passés en revue une expérience ou un événement marquant. II peut faire une rétrospective du progrès accompli, au moment où le groupe envisage de nouvelles avenues ou de nouveaux projets, en présence de visiteurs ou de nouveaux participants, lorsque le groupe semble s'embourber ou dissiper ses énergies, se désintéresser du travail entrepris ou lorsqu'il est près d'atteindre l'étape finale. L'intervenant peut aussi décider de signaler régulièrement les accomplissements du groupe, afin d'aider ce dernier à s'auto-évaluer.

\section{Préserver l'histoire et la continuité du groupe}

L'intervenant peut préserver l'histoire et la continuité du groupe de trois façons : 1) en rappelant au groupe ses expériences antérieures, positives et négatives; 2 ) en faisant délibérément le lien entre la session actuelle et la précédente, ceci en résumant en une phrase ce qui s'est passé la semaine précédente; 3) en aidant le groupe à développer des symboles de ses expériences passées. L'intervenant assure cette continuité en le mentionnant au début d'une session, en faisant périodiquement des liens avec l'histoire passée lorsque le groupe semble disposé à s'engager dans une réflexion, lorsqu'il est dans une impasse, lorsqu'il est dans une phase de planification ou d'évaluation, et toujours lors de la dernière étape de l'expérience de groupe.

\section{Énoncer les normes}

Le travailleur social énonce des normes, comme moyen d'attirer l'attention sur l'esprit de groupe. II demande parfois au groupe de se rappeler une norme ou, de temps en temps, mentionne lui-même la norme établie, de façon à renforcer les règles mises en place par le groupe lui-même. L'intervenant rappelle au groupe certaines normes que les membres semblent oublier ou ignorer, ou lorsque de nouveaux membres ont besoin d'informations sur le fonctionnement du groupe ou d'une explication concernant des actions particulières.

\section{Encourager le développement de traditions et de rituels}

Pour encourager le développement de traditions et de rituels, le travailleur social doit comprendre la valeur des symboles marquant l'identité du groupe, encourager leur expression et suggérer la création de tels symboles, s'ils sont absents. Des rituels peuvent être utilisés pour commencer ou terminer les réunions, lors d'événements 
spéciaux, à la fin d'une série de rencontres ou lors d'une prise de contact avec d'autres groupes, associations ou organismes de la communauté. Les symboles ou les comportements symboliques peuvent faire partie des réunions d'une manière courante et prévisible ou alors servir à rappeler aux membres qu'ils font partie du groupe, $s^{\prime}$ ils ne $s^{\prime}$ impliquent pas lors d'une réunion.

$D^{\prime}$ autres habiletés ponctuelles peuvent être plus particulièrement utilisées pour faciliter le travail du groupe. Nous en retenons onze parmi les principales.

\section{Amplifier des messages subtils}

Amplifier un message subtil, c'est attirer l'attention sur des éléments de communication passés inaperçus - mots, ton de la voix, expressions faciales - en les verbalisant. L'intervenant amplifie un message subtil lorsque les trois conditions suivantes sont réunies : 1) plus de deux personnes sont présentes; 2) le comportement d'une personne est inapproprié dans la situation; par exemple, tout le monde rit sauf une personne qui reste silencieuse et fixe le sol; 3) les autres personnes présentes ne semblent pas remarquer le comportement. II s'agit alors d'attirer l'attention sur le fait qu'un message a été émis. Aussi, l'intervenant devrait adresser son commentaire aux autres, non pas à la personne dont il commente le comportement.

\section{Atténuer l'impact de messages trop puissants}

Atténuer l'impact d'un message trop puissant, c'est verbaliser l'essence d'un message hautement émotif (cris, coups, regards) de façon à réduire la force de l'affect et à rendre le message " audible ". La puissance d'un message devrait être diminuée lorsqu'il apparaît que les membres du groupe ne peuvent l'encaisser à l'intensité ou au registre auxquels il est émis.

\section{Amener le groupe à réagir à un sentiment ou une émotion exprimés}

Amener le groupe à réagir à un sentiment se fait en aidant les membres à examiner leurs propres sentiments et émotions et à les partager avec les autres, face à un sentiment exprimé par l'un d'eux. Cette technique s'applique quand 1) plus de deux personnes sont présentes et 2) un sentiment est exprimé. Dans les cas où un sentiment est exprimé lors d'une rencontre entre l'intervenant et une seule autre personne, l'attitude appropriée consiste à se centrer sur le sentiment. 


\section{Amener le groupe à réagir à un énoncé ou une question}

Amener le groupe à réagir à un énoncé ou une question se fait en demandant aux autres de s'attarder sur l'énoncé ou la question exprimés par un membre et à en discuter. Cette technique s'applique quand 1) plus de deux personnes sont présentes et 2) les autres possèdent vraisemblablement l'information nécessaire pour discuter de la question soulevée.

\section{Rediriger les messages}

Rediriger un message, c'est demander à quelqu'un qui émet un message de diriger cet énoncé ou sentiment directement à la personne à qui i! s'adresse, que cette personne soit présente ou non. Ce comportement est utilisé quand 1) plus de deux personnes sont présentes; 2) une personne dirige son message vers l'intervenant ou vers quelqu'un d'autre tandis que la personne à qui il s'adresse est présente et l'entend; 3) une personne dirige vers l'intervenant un message qui s'adresse à un membre absent. Ceci peut arriver avec l'intervenant seul ou en présence des autres.

\section{Inciter à la pleine participation}

L'intervenant incite à la pleine participation en demandant à certains membres qui ne participent pas de parler, soit en les regardant, soit en suscitant verbalement leurs commentaires. Cette technique peut s'appliquer quand 1) plus de deux personnes sont présentes; 2) certains parlent, d'autres pas; 3) une ou plusieurs personnes semblent dominer la communication.

\section{Remettre les problèmes entre les mains du groupe}

Remettre les problèmes entre les mains du groupe, c'est demander aux participants de faire face à leurs problèmes, spéculations ou projets, à la lumière de leur propre expérience et de leurs réflexions, plutôt que d'éviter de s'attaquer à ces questions. Le travailleur social remet un problème entre les mains du groupe quand 1) il y a plus de deux personnes présentes; 2) le groupe cherche à être conseillé ou dirigé par l'intervenant; 3) il assume que le groupe est en mesure de s'occuper de cette question lui-même. II devrait adopter cette attitude chaque fois que les participants du groupe font appel à lui, à ses connaissances et expériences soi-disant supérieures. Avec le temps, le groupe en viendra à anticiper une telle réaction de l'intervenant et commencera à prendre plus d'initiative pour s'occuper, à sa façon, de ses problèmes et de ses affaires. 


\section{Rechercher le consensus}

Rechercher le consensus, c'est vérifier si la majorité des participants sont d'accord avec la façon dont vont les choses. Le travailleur social devrait vérifier périodiquement s'il y a consensus quand 1) plus de deux personnes sont présentes; 2) la discussion est chaude ou animée; 3) un petit nombre de participants semblent dominer la discussion.

\section{Susciter différents points de vue}

Susciter différents points de vue, c'est aider les participants du groupe à voir les choses sous des angles différents, à passer en revue diverses possibilités. Si le groupe n'exprime que des points positifs, le travailleur social devrait faire émerger le point de vue contraire. Mais s'il ne considère que les côtés négatifs, alors l'intervenant cherchera à lui faire exprimer d'autres possibilités. L'intervenant aide en outre le groupe à voir et à réfléchir au-delà d'une pensée dichotomique : bien/mal, oui/non, bon/mauvais. Autrement dit, la réflexion et l'action peuvent éventuellement comporter des nuances et une variété de choix et d'options qui déboucheront sur un grand nombre de solutions possibles. Susciter différents points de vue, c'est un moyen de lutter contre I'homogénéité d'une pensée de groupe (groupthink), c'est-à-dire une tendance des participants à rechercher la cohésion et I'entente à tout prix, face aux pressions de groupe vers la conformité et l'efficacité. Cette homogénéité masque la richesse d'une réflexion diversifiée.

\section{Provoquer de nouvelles formes d'expression}

Quelquefois, des activités sont valables simplement parce qu'elles incitent le groupe à une action qui ne se serait pas produite autrement. Un élément " magique » dans l'activité a aidé l'intervenant et le groupe à entamer ensemble un processus de travail : il peut s'agir du caractère ludique de l'activité, du lien qu'elle suggère avec des temps meilleurs, du changement qu'elle apporte par rapport aux affaires sérieuses et aux soucis, ou encore de l'humour qu'elle suscite, de l'appel à la spontanéité, de la juxtaposition tout à fait inattendue d'éléments différents dans une situation, ou tout ceci à la fois.

Quelles qu'en soient les raisons, les activités proposées d'une façon réfléchie comme étant potentiellement intéressantes (et appropriées à l'âge, à la classe, à la culture et au sexe des participants) peuvent mener à des domaines inexploités et transporter le groupe et 
ses participants dans un espace nouveau, différent. Et au cours de cette expérience, de nouvelles "voix » peuvent s'exprimer qui ne se sont pas fait entendre auparavant.

\section{Construire sur la base des forces individuelles}

Si nous adoptons une vision globale de l'individu et regardons au-delà de la personne-qui-a-un-problème pour voir tout ce que la personne sait et peut faire, de nouvelles perspectives nous apparaissent. Les "laissés pour compte ", les " difficiles d'accès » (" hard-toreach "), les sans-abri, le parent unique en difficulté, l'adolescente célibataire qui a deux enfants, tous ces gens ont quelque chose en eux qui demande à être apprécié. Ils doivent avoir des forces pour survivre comme ils le font dans les situations difficiles auxquelles ils font face. Que savent-ils ? Que peuvent-ils nous montrer si nous leur en donnons la chance?

\section{Observation des habiletés de groupe ${ }^{2}$}

La présente feuille de pointage sert à apprécier les habiletés d'intervention, pour une période donnée, dans un groupe.

Les habiletés qui suivent devraient se retrouver de façon constante dans tout groupe :

1. Penser en termes de groupe.

2. Balayer du regard (scanning).

3. Favoriser la cohésion.

Les habiletés suivantes sont plus ponctuelles. Cochez à mesure que vous les observez :

\section{Pour la formation du groupe}

4. Choisir délibérément des modèles de communication.

5. Signaler les réalisations du groupe.

6. Préserver I'histoire et la continuité du groupe.

7. Énoncer les normes.

8. Encourager le développement de traditions et de rituels.

\section{Pour faciliter le travail du groupe}

9. Amplifier des messages subtils.

10. Atténuer l'impact de messages trop puissants.

11. Amener le groupe à réagir à un sentiment ou une émotion exprimés. 
12. Amener le groupe à réagir à un énoncé ou une question.

13. Rediriger les messages.

14. Inciter à la pleine participation.

15. Remettre les problèmes entre les mains du groupe.

16. Rechercher le consensus.

17. Susciter différents points de vue.

18. Provoquer de nouvelles formes d'expression.

19. Construire sur la base des forces individuelles.

\section{Notes}

${ }^{1}$ Ce texte a été présenté le 28 octobre 1989, lors du $11^{\mathrm{e}}$ symposium annuel sur le service social des groupes, dans le cadre d'une "classe de maître ", sous le titre de "Skills for working with Groups at the Group Level ". On peut obtenir des informations plus complètes en consultant l'ouvrage de Ruth R. Middleman et Gale Goldberg Wood : Skills for Direct Practice in Social Work. New York : Columbia University Press (sous presse). Le présent article a été traduit par Lyne Champoux.

${ }^{2}$ Tiré de Ruth R. Middleman et Gale Goldberg Wood, op. cit. 